\title{
EEG-based decoding and recognition of imagined music
}

Marthe Tibo, Simon Geirnaert, Alexander Bertrand

The authors have withdrawn their manuscript because they discovered an error in the analysis code after publication of the preprint, which turns out to have a major impact on the main results in the paper. The results on the imagination data become non-significant after correcting for the mistake. Significant results on the perception data are preserved, although the classification results are worse than what is reported.

Therefore, the authors do not wish this work to be cited as a reference.

If you have any questions, please contact the last author. 